\title{
Pitfalls in the diagnosis of acoustic neuroma
}

\author{
L. M. FLOOD, R. E. BRAMMER, M. D. GRAHAM AND \\ J, L. KEMINK \\ Department of Otolaryngology, Head and Neck Surgery. University of Michigan, Ann Arbor,
} Michigan, USA

Accepted for publication 4 April 1984

Flood L. M., Brammer R. E., Graham M. D. \& Kemink J. L. (1984) Clin. Otolaryngol. 9, 165-170

Pitfalls in the diagnosis of acoustic neuroma

The patient with an acoustic neuroma may present to the otologist with a variety of clinical features. Classically these include a retrocochlear pattern of sensorineural hearing loss, reduced vestibular response on caloric testing and radiological asymmetry of the internal auditory canals (IAC). The absence of any or all of these features, however, does not exclude the presence of tumour. Five cases are presented to illustrate the potential for diagnostic delay unless a routine battery of investigations is undertaken in patients with neuro-otological disorders. All patterns of subjective audiometry are encountered, auditory brain stem response testing may be unreliable where hearing loss is profound, vestibular testing is normal in half of small tumours and the intracanalicular tumour may be radiologically undetectable unless IAC meatography is employed.

Keywords neuroma acoustic

Despite increasingly sophisticated techniques of investigation to identify patients with acoustic neuroma, these tumours are commonly advanced at presentation. A delay in initial diagnosis increases the morbidity of subsequent surgery and may be of medico-legal significance. Five patients seen at the Department of Otolaryngology-Head and Neck Surgery, University of Michigan, are presented to illustrate some pitfalls that may be encountered in the diagnosis or exclusion of acoustic neuroma.

CASE 1

RS. A 36-year-old man first sought specialist advice in 1979 following a sudden onset of right high frequency hearing loss. Tomography of the internal auditory canals (IAC) was normal and no further investigation was undertaken. Two years later the hearing loss had progressed (SRT $10 \mathrm{~dB}$, s.d. $76 \%$ ) with worsening tinnitus. A CT scan was reported normal. On auditory brain stem response (ABR) testing, although wave I was grossly delayed, interwave latencies were normal.

Six months later a further deterioration in hearing prompted his first referral to this hospital. There was no speech discrimination on the right side and again ABR testing showed delay of all absolute latencies but normal interwave intervals. ENG testing showed an RVR of $27 \%$ on the right. Computerized tomography (CT)

Correspondence: Dr Liam M. Flood, Senior Registrar, Royal Ear Hospital, University College Hospital, Capper Street, London WC1E 6AIJ, UK. 
scanning showed slight IAC asymmetry with some fluting on the right, but no lesion was evident with contrast intravenously. However, pantopaque ${ }^{\circledR}$ myelography demonstrated a $1 \mathrm{~cm}$ intracanalicular tumour, which was excised via a translabyrinthine approach.

Comment This small tumour produced no radiological evidence of bony erosion at presentation and being intracanalicular was not evident on CT even with enhancement. ENG showed only a mild RVR. Only repeat CT scanning offered any suggestion of the underlying diagnosis and justified myelography. The small intracanalicular tumour, the quest of all neurootologists, produces few clinical features.

\section{CASE 2}

JL. A 54-year-old man presented to an otolaryngologist in 1973 with bilateral conductive hearing loss due to longstanding chronic suppurative otitis media. Tympanoplasty on the right, the worse hearing ear, produced no hearing gain. In 1977 he noticed sudden onset of a left hearing loss. Audiometry showed a mixed loss (SRT 35, speech discrimination $44 \%$ ). Plain films of the petrous pyramids were normal and Bekesy audiometry suggested a cochlear pattern of loss. The hearing on the left deteriorated until referral in 1983 when audiometry showed symmetric thresholds (SRT right $50 \mathrm{~dB}$, SRT left $65 \mathrm{~dB}$ ) but a marked difference in discrimination (SD right $84 \%$, left $8 \%$ ). ABR testing showed gross delay in both absolute latencies and I-V interval on the left, even when corrected for conductive and sensorineural loss. However, brisk responses were obtained to caloric testing in both ears. CT scanning was normal but meatography demonstrated a $1 \mathrm{~cm}$ filling defect within the left IAC. The general medical condition of the patient precluded surgery.

Comment Even in the presence of established pathology, sensorineural hearing loss may indicate an acoustic neuroma. While progressive cochlear deafness may occur in chronic suppurative otitis media, the asymmetry of speech discrimination suggested retrocochlear involvement. The long history and small size of tumour at ultimate diagnosis suggest slow growth.

\section{CASE 3}

AS. A 48-year-old man complained of a 7 year history of progressive left sided deafness. There was a history of considerable industrial noise exposure but no other relevant features. He did not complain of imbalance or vertigo but noted a ringing tinnitus in the left ear. Investigation elsewhere confirmed a profound sensorineural hearing loss, normal vestibular investigations and no canal asymmetry on computerized tomography. On presentation to this hospital after a 2 year interval pure tone audiometry showed a flat 80-90 dB threshold with no speech discrimination. Caloric irrigation produced symmetrical responses but $\mathrm{CT}$ showed a $1 \mathrm{~cm}$ enhancing lesion within the left IAC, with minimal extension into the cerebellopontine angle.

Comment The profound hearing loss prevented identification of retrocochlear pathology by auditory brain stem response (ABR). Again, a small tumour produced both a normal ENG and being intracanalicular, a normal CT scan. Only repeat of baseline investigations and CT scan, after a 2 year interval alerted the investigators to the diagnosis.

\section{CASE 4}

PK. A 54-year-old woman presented with a 2 year history of bilateral tinnitus and a sensation of fullness in the right ear. There was no complaint of dysequilibrium or hearing loss. Examination suggested some hypoaesthesia of the right face. A pure tone audiogram was symmetric with normal 
thresholds but speech discrimination showed slight impairment (right SDT $86 \%$, left SDT 96\%).

However, an ENG showed a right 100\% RVR and ABR testing demonstrated a $1 \mathrm{~ms}$ delay in $\mathrm{I}-\mathrm{V}$ interwave latencies with reduced amplitude of waves IV and $\mathrm{V}$ on the right. CT scanning revealed a $2 \mathrm{~cm}$ enhancing lesion within the right cerebellopontine angle. This was removed via a translabyrinthine approach and histological examination confirmed an acoustic neuroma.

Comment The presenting complaint of bilateral tinnitus and normal pure tone audiometry did not immediately suggest the diagnosis However, the mild asymmetry in discrimination indicated retrocochlear pathology. The complete canal paresis with no history of subjective vertigo was highly suggestive of an acoustic neuroma. ABR testing and CT scanning again proved reliable diagnostic tests.

\section{CASE 5}

HK. a 33-year-old woman presented in 1983 with a 9 year history of left sided hearing loss. Deafness had progressed following a sudden onset during labour though investigation failed to provide a diagnosis. Latterly she complained of deep otalgia and mild dysequilibrium. There was no recordable hearing on the left on audiometry or ABR and ENG demonstrated a complete left canal paresis. Plain $\mathrm{X}$-rays of the IAC were compromized by pneumatization but were reported normal. However, a CT scan demonstrated a large mass in the cerebellopontine angle and she is awaiting resection using a planned 2stage combined translabyrinthine and suboccipital approach.

Comment The sudden onset of deafness in a young woman arising during delivery suggested a vascular or labyrinthine membrane lesion rather than the diagnosis of acoustic neuroma. There are no details of initial investigation but even after 9 years the IAC plain films were reported normal suggesting the tumour arose within the cerebellopontine angle.

\section{Discussion}

Unfortunately most neuro-otologists will be familiar with such cases, where some nonclassical feature in presentation has led to diagnostic confusion. Cushing ${ }^{1}$ described a progression of clinical features as the acoustic neuroma expands. Usually commencing at the neurilemmal-neuroglial junction of the superior vestibular nerve, it grows from the IAC into the cerebellopontine angle. $\mathrm{He}$ found a sequence of auditory and vestibular disturbance, headache, inco-ordination and ataxia, involvement of adjacent cranial nerves (producing facial sensory and motor loss), raised intracranial pressure, dysarthria, dysphagia and cerebellar crises in all but $20 \%$ of his cases. Alterations in this progression are encountered. One-third of tumours, especially those arising more medially, may present with neurological symptoms rather than cochleovestibular disorders. ${ }^{2}$ Presentation varies with the site of the tumour, along the cochleovestibular nerve, from the posterior fossa to within the labyrinth itself. ${ }^{3}$

The majority of acoustic neuromas are minute, asymptomatic and so are never diagnosed during life. Stewart et al. ${ }^{4}$ demonstrated 5 occult vestibular schwannomas in 517 post-mortem examinations, consistent with Morrison's description of a $1 \%$ incidence of symptomless tumours at death, while the clinical incidence is only 1 in 100,000 of the population. ${ }^{5}$

Clemis states that 'every progressive asymmetric sensorineural hearing loss is due to an acoustic tumour until proven otherwise. ${ }^{6}$ However, the yield from investigation will be limited since only $5-10 \%$ of patients with such symptomatology will have a neuroma. ${ }^{7}$ Nonetheless, the goal remains as diagnosis of the smaller relatively asymptomatic tumour to 
prevent brain stem and cerebellar involvement, improve the prognosis for facial nerve preservation at surgery and ultimately allow conservation of hearing in some cases. ${ }^{8}$

Normal or symmetrical hearing is to be expected in $5 \%$ of acoustic neuromas, although a high frequency loss is the most common finding. ${ }^{9}$ Inconsistencies on audiometry reflect the difficulties of the neuroma patient in determining reliable thresholds with contralateral masking and should not be ascribed to a fluctuating hearing loss. Schuknecht \& Woellner ${ }^{10}$ have shown that normal pure tone thresholds are preserved for any frequency while only $25 \%$ of the ganglion cells for that frequency survive. However, the complex auditory signals of speech demand greater nerve survival and so disproportionately poor speech discrimination is to be found in $70 \%$ of cases. ${ }^{11}$ In a series of 500 cases, only $28 \%$ achieved scores in excess of $60 \% .^{12}$

The small proximally placed tumour with a flat audiometric trace is especially liable to show atypical features on further subjective audiometry. Only two-thirds of acoustic neuromas produce a retrocochlear pattern of deafness. ${ }^{13}$ Thomsen et al. found recruitment as shown on ABLB testing in 4 of 59 tumour cases. ${ }^{14}$ Abnormalities of stapedial reflex threshold or decay may be detected in up to $98 \%$ of such patients although false positive results occur. ${ }^{15}$ Acoustic reflexes will be abolished even by cochlear hearing losses of greater than $60-75 \mathrm{~dB}^{16}$ Abnormal auditory adaption as shown by the Cahart test is not evident in up to one-third of tumour cases. ${ }^{7,12}$

Electric response audiometry has proven a more reliable indicator of rectrocochlear pathology than psychoacoustical testing and has largely superseded it. In isolation transtympanic electrocochleography is of little value in excluding tumours ${ }^{17,18}$ but may exhibit (a) a distorted widened action potential, (b) a discrepancy between AP threshold and that of the cochlear micro- phonic or subjective hearing, (c) a shift in latency amplitude function. ${ }^{19}$ Eggermont et al. ${ }^{18}$ have demonstrated that such a retrocochlear picture is only shown by hearing losses in excess of $60 \mathrm{~dB}$. Milder hearing losses due to acoustic neuroma are purely cochlear. This misleading end organ pattern of deafness may be the result of hair cell degeneration due to compression of the nutrient internal auditory artery, toxic protein concentration in perilymph, direct cochlear invasion or involvement of efferent neurons. ${ }^{20}$

The ABR evaluates conduction of the auditory signal up to the inferior colliculus and can detect up to $98 \%$ of cerebellopontine angle lesions ${ }^{21}$ and predict tumour size. ${ }^{18}$ Indications of retrocochlear pathology include: increased wave $\mathrm{V}$ latency, increased wave I-V interval or interaural latency difference greater than $0.2 \mathrm{~ms}$. Care must be taken in the analysis of ABR findings in the presence of a conductive loss or a high frequency loss greater than $70 \mathrm{~dB}$. Where no $\mathrm{ABR}$ is obtained but wave $I$ is preserved on electrocochleography, a brain stem or cerebellopontine angle tumour is indicated. Brackmann \& Selters have calculated adjustments of the wave I-V interval to allow for high frequency losses. ${ }^{22}$ For every $10 \mathrm{~dB}$ loss above $50 \mathrm{~dB}$ at $4 \mathrm{KHz}$ they subtract $0.1 \mathrm{~ms}$ from the $\mathrm{V}$ wave latency. While increasing the sensitivity of ABR testing $3.2 \%$ of acoustic neuromas still produce normal results. In a patient presenting with purely neurological symptoms a normal ABR reliably excludes neuroma as only larger tumours involve the cranial nerves, brain stem and cerebellum. ${ }^{2}$

Vestibular testing is a poor screening test for acoustic neuroma. Pulec \& House ${ }^{23}$ found a symmetrical response to caloric irrigation in only $4 \%$ of their series. A reduced vestibular response of greater than $30 \%$ is to be expected in 9 out of 10 of large tumours but occurs in fewer than half of smaller neuromas. ${ }^{24}$

Histopathological study suggests that the normal ENG is found chiefly in smaller 
neuromas arising from the inferior vestibular nerve. ${ }^{25}$ The inferior nerve is not evaluated by caloric testing of the lateral semicircular canal.

Due to the gradual loss of vestibular function and consequent central compensation, major subjective vertigo is not a usual feature of acoustic neuroma. Therefore, a major reduction in canal function on ENG testing without corresponding vestibular symptomatology is highly suggestive of an VIIIth nerve tumour. In contrast, 151 patients with unilateral Meniere's disease investigated between attacks, showed a mean RVR of only $34.3 \%{ }^{26}$ Central patterns of nystagmus imply brain stem compression with impaired function of the vestibular nuclei, cerebellar peduncles and cerebellum.

Definite diagnosis of IAC disease relies on radiological evidence of bony erosion, demonstration of an enhancing lesion using CT scan or ultimate proof of a space occupying lesion within the canal on meatography. Plain radiographic views of the IACs are compromised by superimposed pneumatization or bony structures. ${ }^{27}$ In some series only half of the tumours examined are detected. ${ }^{13}$ However, Crabtree and House found radiological evidence of canal erosion and expansion in $85 \%$ of tumour patients employing Stenver, Towne, lateral and transorbital views.

Polytomography is superior to plain $\mathrm{X}$ rays when pneumatization of the petrous pyramid obscures the canals. ${ }^{28}$ An acoustic neuroma is suggested by erosion of the posterior lip of the IAC, asymmetry greater than $2 \mathrm{~mm}$, thinning of the superior bony wall, shortening of the posterior wall greater than $3 \mathrm{~mm}$, abnormalities which are found in $80 \%$ of acoustic neuromas. ${ }^{29}$ In a series of 59 tumour patients Thomsen et al. found symmetrical IACs on tomography in $10 \% .^{30}$ Normal petrous films in the presence of a cerebellopontine angle syndrome suggest extracanalicular origin of a vestibular schwannoma or a meningioma.
Computerized tomography combined with intravenous contrast enhancement will detect $90 \%$ of tumours as small as $1.5 \mathrm{~cm}$ in diameter. ${ }^{31}$ While extension of as little as $5 \mathrm{~mm}$ into the posterior fossa is reliably detected, intracanalicular tumours can be missed. Cisternography must be employed where clinical suspicion warrants. Difficulty may be encountered in admitting air or $\mathrm{CO}_{2}$ into a normal IAC falsely suggesting a space occupying lesion. Pantopaque ${ }^{\circledR}$ myelography is preferable in this regard. As in all the previous investigations, the possibility of bilateral acoustic neuromas must be considered and the contralateral IAC evaluated.

\section{Summary}

The myriad presentations and clinical features of acoustic neuroma potentially carry many pitfalls for the otologist.

(I) Pure tone audiometry may be normal or any hearing loss symmetrical.

(II) The psychoacoustic test battery, e.g. tone decay, recruitment, discrimination may suggest a cochlear disorder.

(III) Electrocochleography suggests a cochlear pattern of deafness up to $60 \mathrm{~dB}$ losses. ABR may be unreliable in the presence of marked or high frequency hearing loss although the corrective methods described contribute much to reliability.

(IV) Small tumours are frequently associated with a symmetrical caloric response.

(V) Plain radiographs of the IAC may be difficult to interpret where the temporal bone is well pneumatized. Where the tumour is intracanalicular only myelography may reveal it.

(VI) Progressive sensorineural loss may be overlooked when associated with other pre-existing aural pathologies. Acoustic neuroma has been described complicating established Meniere's disease, otosclerosis and noise exposure. ${ }^{32-34}$ 
Baseline investigation of patients with hearing loss, dysequilibrium or tinnitus should include audiometry, ABR, ENG and plain X-rays of the petrous pyramids. None of these, in isolation, excludes neuroma and investigation will need repetition at intervals to monitor any progression. CT scanning with enhancement may not detect the smaller intracanalicular lesions, only myelography can exclude these. The mainstay of diagnosis remains a high index of suspicion on the part of the investigator and undue reliance can be placed on no single test.

\section{References}

1 Cushing H. (1917) Tumours of the nervus acousticus and the syndrome of the cerebellopontine angle. W.B. Saunders, Philadelphia

2 Hart R.G., Gardiner D.P. \& Howieson J. (1983) Acoustic tumors: atypical features and recent diagnostic tests. Neurology 33, 211-221

3 Hawke M., Keene M. \& Robbins K.T. (1981) Intralabyrinthine schwannoma. $J$. Otolaryngol. 10, 313-320

4 Stewart T.J., Liland J. \& Schuknecht H.F. (1975) Occult Schwannomas of the vestibular nerve. Arch. Otolaryngol. 101, 91-95

5 MORRISON A.W. (1975) In Management of sensorineural deafness. pp. 46-79. Butterworths, London

6 CLEMIS J.D. (1982) In Neurological surgery of the ear and skull base (ed.) D.E. Brackman, pp. 207-212. Raven Press, New York

7 JohNSON E.W. (1977) Auditory test results in 500 cases of acoustic neuroma. Arch. Otolaryngol. 103, 152-158

8 RAND R.W. \& Kurze T. (1968) Preservation of vestibular, cochlear and facial nerves during microsurgical removal of acoustic tumors. $J$. Neurosurg. 28, 158-161

9 Hart R.G. \& DavenPort J. (1981) Diagnosis of acoustic neuroma. Neurosurgery $9,450-463$

10 Schuknecht H.F. \& Woellner R.C. (1955) An experimental and clinical study of deafness from lesions of the cochlear nerve. J. Laryngol. Otol. 69, 75-97

11 Canty P. (1978) Speech audiometry in acoustic neuroma. J. Laryngol. Otol. 92, 843-851

12 Mathew G.D., Facer G.W., Suh K.W., et al. (1978) Symptoms, findings and methods of diagnosis in patients with acoustic neuroma. Laryngoscope, 88, 1893-1903

13 Singh K.P., SMYTH G.D.L. \& Gordon D.S. (1978) The diagnosis of acoustic neuroma. $J$ Laryngol. Otol. 92, 1-7

14 Thomsen J., Terkildosen K. \& Tos M. (1983) Acoustic neuromas progression of hearing impair- ment and function of the eighth cranial nerve. Am. J. Otol. 5, 21-33

15 HirsCh A. \& ANDERSON H. (1980) Audiological test results in 96 patients with tumors affecting the eighth nerve. Acta Otolaryngol. (Suppl) 369, 1-26

16 Musiek F.E., Mueller R.J., KibBe K.S. \& RaCKLIfFe L.M. (1983) Audiologic test selection in the detection of eighth nerve disorders. $A m$. $J$. Otol. 4, 281-287

17 Clemis J.D. \& Mitchell C. (1977) Electrocochleography and brainstem responses used in the diagnosis of acoustic tumors. J. Otolaryngol. 102, 654

18 EgGermont J.J., Don M. \& Brackmann D.E. (1980) Electrocochleography and auditory brain stem electric responses in patients with pontine angle tumours. Ann. Otolaryngol. (Suppl) 1-18

19 Portman M., Cazals Y., Negrevergne M. \& ARAN J.-M. (1980) Transtympanic and surface recordings in the diagnosis of retrocochlear disorders. Acta Otolaryngol. 89, 362-369

20 Flood L.M. \& Brightwell A.P. (1984) Cochlear deafness in the presentation of a large acoustic neuroma. $J$. Laryngol. Otol. 98, 87-92

2.1 GlassCOCK M.E., JACKSON C.G., JOSEY A.F. et al. (1979) Brain stem evoked response: audiometry in clinical practice. Laryngoscope 89, 1021-1035

22 Brackmann D.E. \& Selters W.A. (1982) In Neurological surgery of the ear and skull base (ed.) D.E. Brackmann, Raven Press, New York

23 Pulec J.L. \& House W.G. (1964) Vestibular involvement and testing in acoustic neuromas. Arch. Otolaryngol. 80, 677-681

24 Linthicum F.H. \& ChURChill D. (1968) Vestibular test results in acoustic tumour cases. Arch. Otolaryngol. 88, 604-609

25 Neely J.G., Britton B.H. \& Greenberg S.D. (1976) Microscopic characteristics of the acoustic tumor in relationship of its nerve of origin. Laryngoscope 86, 984-991

26 HulshoF J.H. \& BAARSMA E.A. (1981) Vestibular investigation in Meniere's disease. Acta Otolaryngol. 91, 75-81

27 Crabtree, J.A. \& House W.F. (1964) X-ray diagnosis of acoustic neuromas. Arch. Otolaryngol. 80, 695-697

28 Glasscock M.E., Overfield R.E., \& Miller G.W. (1976) Polytomography in otological practice. South. Med. J. 69, 1433-1437

29 Harner S.G. \& Laws E.R. (1981) Diagnosis of acoustic neuroma. Neurosurgery 9, 373-379

30 Thomsen J., Reiter S., BorUm P. et al. (1981) Tomography of the internal auditory meatus. $J$. Laryngol. Otol. 95, 1191-1204

31 HaNAFEE W.N. (1981) Computerized tomography in hearing loss and dysequilibrium states. Otol. $H$. N. Surgery 89, 861-866

32 DeMoura L.F. (1968) In Discussion in Meniere's disease (ed.) J. Pulec. p. 347. W.B. Saunders, Philadelphia.

33 Drake-Lee A. (1981) Late onset sensorineural hearing loss following stapedectomy. $J$. Laryngol. Otol. 95, 739-744

34 Miller M.H., Doyle T.J. \& Geier S.R. (1981) Acoustic neuroma in a population of noise exposed workers. Laryngoscope 91, 363-371 